\title{
Correction of Class II Division 2 Deepbite Malocclusion with Non-extraction Therapy
}

\author{
Dr Situ L Shrestha, ${ }^{1}$ Dr Supreeth S Manipal,, Dr Bikash V Shrestha, ${ }^{3}$ Dr Alok K Jaiswal ${ }^{4}$ \\ 'Professor, 2.3Asst Professor, Dept of Orthodontics, Peoples Dental College, Kathmandu, Nepal \\ ${ }^{4}$ Asst Professor, Kedia Dental College, Birgunj, Nepal

\section{ABSTRACT}

The article presents a case report of a teen age girl with Class II Division 2 malocclusion with deep over bite. The case was treated on non-extraction basis using 0.018 pre-adjusted edgewise appliance with anterior bite plate (monobloc) to correct deep bite and forward placement of the mandible. Use of Class II elastics helped to achieve Class I canine and molar retention. Treatment was completed in 22 months with good occlusion and facial esthetics.

\section{INTRODUCTION}

Class II malocclusion is among the common malocclusions that is faced in orthodontic practice. Early diagnosis and proper management can avoid the unnecessary extractions and even orthognathic surgeries that might be needed in some cases. Monobloc (anterior bite plane) appliance can be used in such case to treat the growing skeletal malocclusion. Further treatment refinement is done using Class ॥ elastics, which shows desirable mandibular growth leading to non-extraction correction of skeletal and dental Class II malocclusion.

\section{CASE HISTORY}

A 13 years and 7 months old female patient whose chief complaint in her own words was "I don't like the way my upper teeth are" was presented to the Department of Orthodontics, Peoples Dental College and Hospital. The patient's medical history was noncontributory and her dental history included routine dental check-ups. Other findings and history were unremarkable and perverse habits were not present.

\section{CLINICAL EXAMINATION}

Extra-oral examination of the patient showed symmetric and leptoprosopic facial type on frontal view. The profile view revealed prominent chin, convex profile, prominent upper lip, deep labio-mental sulcus, average nasolabial angle, and competent lip.
Temporomandibular joint examinations confirmed normal joints and maximum inter-incisal opening was acceptable with no deviation. No joint noises were perceptible in excursive movements of the mandible.

Orthodontically the patient presented with Angle's Class II Division 2 malocclusion. Complete traumatic deep bite with $2 \mathrm{~mm}$ overjet and flared upper lateral incisors was noted. There was mild lower anterior crowding (2 $\mathrm{mm})$ and mild upper anterior crowding $(1.5 \mathrm{~mm})$; and a moderate curve of Spee in both arches. The soft tissue was within normal limits (Figure 1).

\section{CEPHALOMETRIC EVALUATION}

Cephalometric analysis (Figure 2) revealed normognathic maxilla (SNA $82^{\circ}$ ), rethrognathic mandible (SNB 73); skeletal Class II relationship (ANB $\left.9^{\circ}\right)$ with a horizontal growth pattern ( $Y$-axis growth $54^{\circ}$ ). The upper and lower central incisors were retroclined (UI to NA angle $6^{\circ}$, LI to NB angle $8^{\circ}$ ), and the chin was prominent (Table I). Panoramic evaluation revealed permanent dentition with all permanent teeth present. The periodontal condition was within normal limits (Figure 3).

\section{TREATMENT}

After reviewing the diagnostic records and patient history; non-extraction orthodontic correction with 
Table 1: Comparative cephalometric analysis

\begin{tabular}{|c|c|c|c|}
\hline Parameters & Pre treatment & Post treatment & Mean value \\
\hline SNA & 78 & 83 & 83.2 \\
\hline SNB & 73 & 78 & 79.8 \\
\hline ANB & 5 & 5 & 3.4 \\
\hline UI to NA & 6 & 21 & 21.3 \\
\hline LI to NB & 8 & 30 & 26.3 \\
\hline Facial Angle & 90 & 87 & 89.8 \\
\hline Angle of Convexity & 4 & 4 & 3,1 \\
\hline Cant of OP & 4 & 4 & 7.1 \\
\hline MP angle & 16 & 20 & 23.2 \\
\hline Y-axis & 54 & 57 & 58.6 \\
\hline
\end{tabular}

preadjusted edgewise appliance technique was initiated, expecting that growth of the mandible would contribute to achieve a Class I molar and canine relationship.

The upper arch was initially banded on the first molars and bonded from second premolar to second premolar with standard Roth prescribed 0.018 pre-adjusted edgewise brackets, with arch wire progression starting from 0.012 NiTi. Mono-block (anterior bite plane) was fabricated and inserted which helped in advancement of mandible. It also acted as anterior bite plate for correction of deep bite. After 1 month lower arch was also bonded and banded.

Class II elastics were used during the treatment and Class I molar and canine relationships were achieved with excellent patient cooperation. After 22 months of active therapy followed by 6 months of active retention period, debonding was done and impressions were made for retainers.

\section{RETENTION}

Clear retainer was placed in maxillary arch and fixed lingual retainer was placed in the mandibular arch. The patient was instructed to wear them full time for 1 year, at night for an additional year, and to return for periodic evaluation until completion of growth.

\section{RESULTS ACHIEVED}

Post-treatment facial photographs are shown in Figure 4. A Class I molar and canine relationship were obtained.
The overbite and overjet were corrected; and maxillary and mandibular crowding was eliminated. The final cephalometric radiograph is shown in Figure 5. The posterior intercuspation was excellent with occlusal settlement and the panoramic radiograph (Figure 6) showed near parallel root position. The maxilla showed a slight forward movement, most likely because of the residual growth. The mandible moved in a favorable forward and downward direction. The upper and lower incisors showed excellent inter-arch relationship. Slight extrusion and significant protraction of the lower molars were observed (Figure 4). This can be attributed to Class II mechanics that opened the bite. The following esthetic changes were achieved: a decrease in facial convexity, increase in anterior facial height, and flatter labiomental sulcus. The profile was well balanced.

\section{DISCUSSION}

The treatment attempted to potentiate more forward growth and development of the mandible. Use of mono-block (anterior bite plane) worked for anterior placement of mandible as well as for correction of deep bite; also to eliminate crowding, correct midline discrepancy, obtain good torque and root axial inclination. Obtaining Class I molar and canine relationship; obtaining an acceptable overjet and overbite; achieve proper intercuspation; improve facial profile by reducing facial convexity and increasing anterior lower facial height were other treatment goals achieved. 

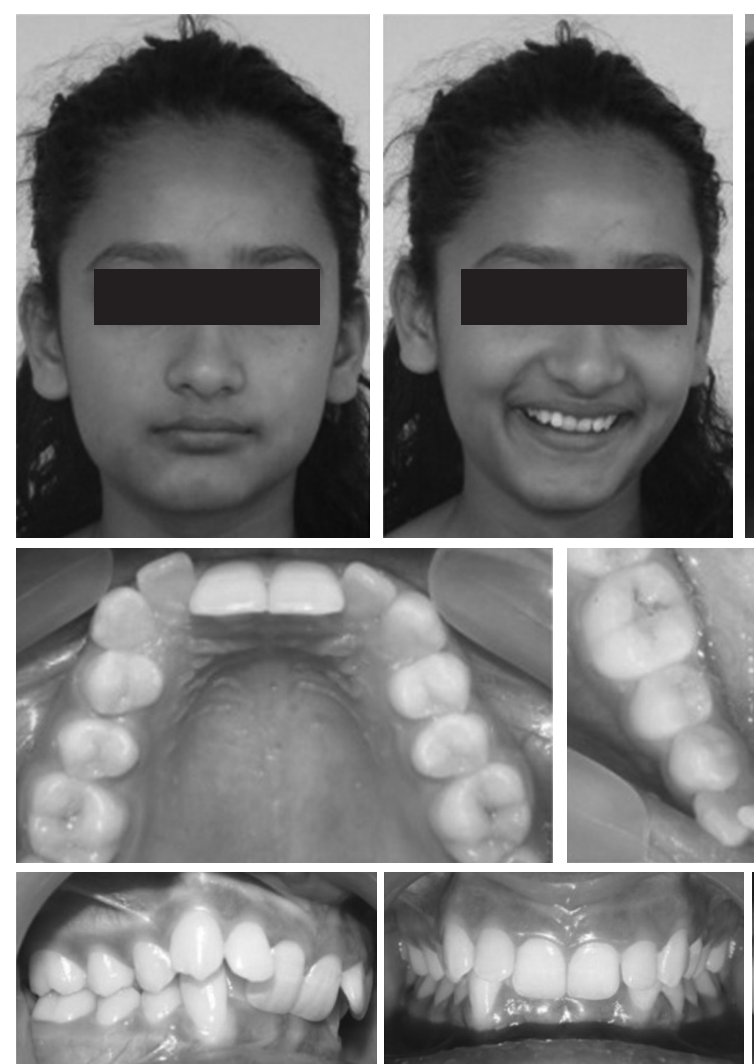

Figure 1: Pretreatment facial and intra-oral photographs
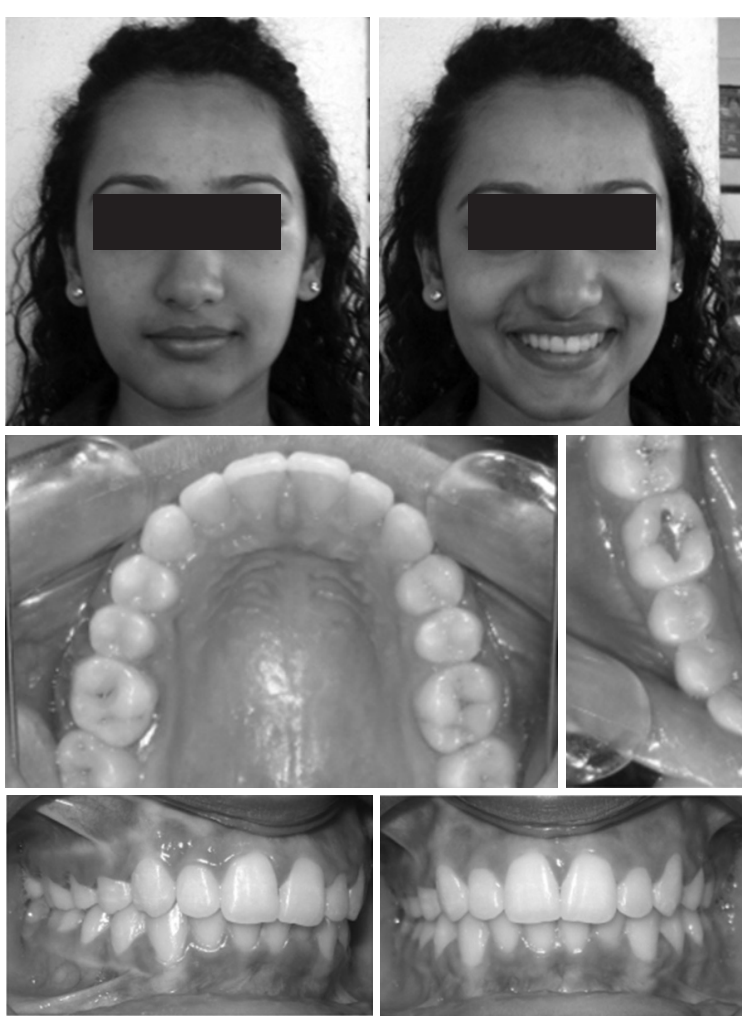

Figure 4: Post treatment facial and intra oral photograph
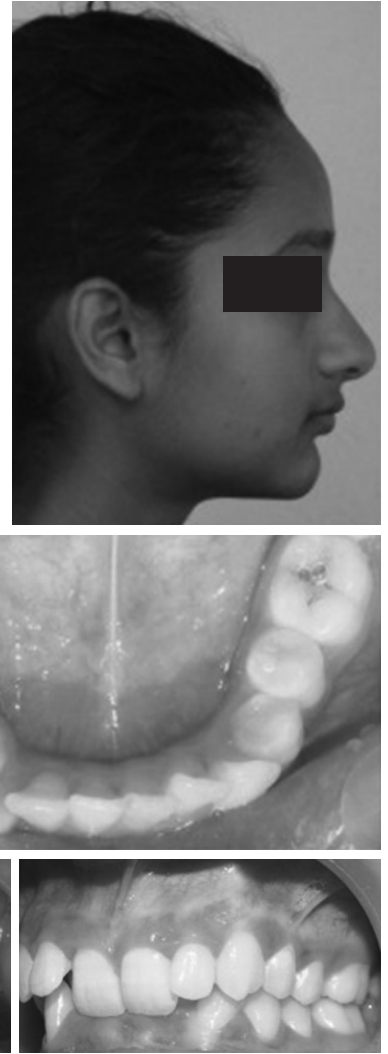

Figure 3: Pretreatment OPG

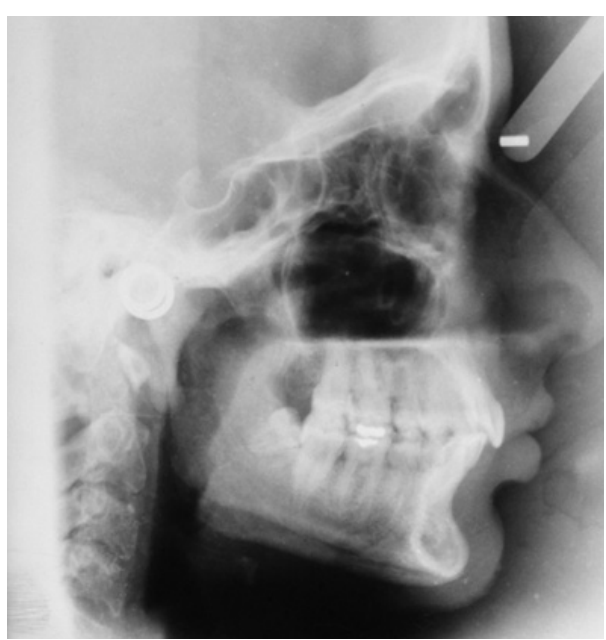

Figure 5: Post treatment Lateral Cephalogram

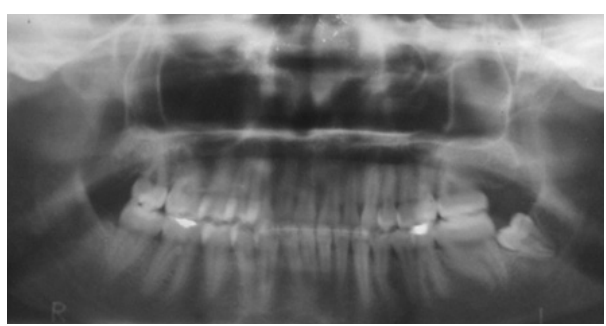

Figure 6: Post treatment OPG
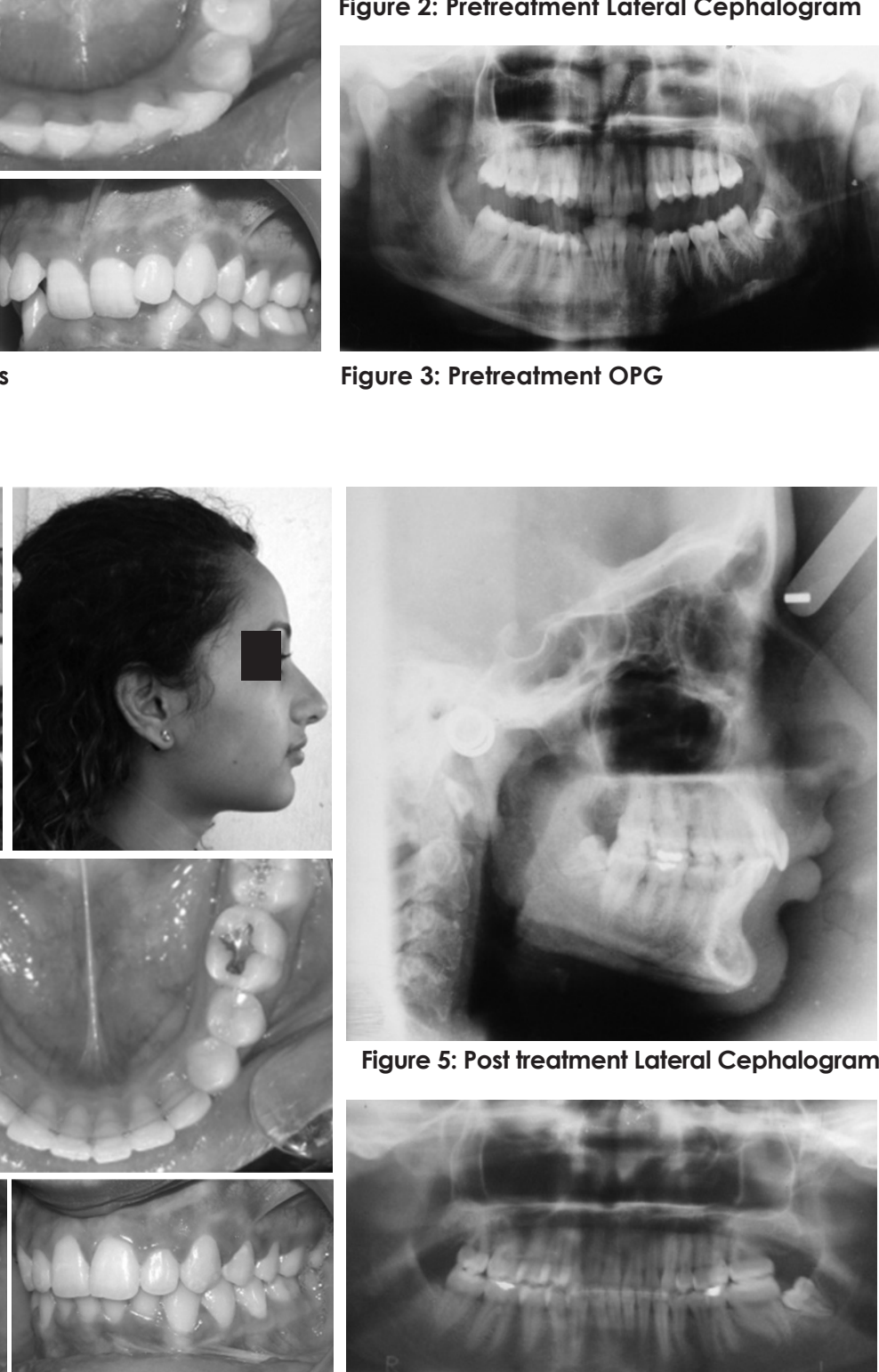